\title{
Basic Fibroblast Growth Factor Modulates Density of Blood Vessels and Preserves Tight Junctions in Organotypic Cortical Cultures of Mice: A New In Vitro Model of the Blood-Brain Barrier
}

\author{
Kerstin Bendfeldt, ${ }^{1}$ Vesna Radojevic, ${ }^{2}$ Josef Kapfhammer, ${ }^{2}$ and Cordula Nitsch ${ }^{1}$ \\ ${ }^{1}$ Section of Neuroanatomy and ${ }^{2}$ Section of Neurodevelopment, Institute of Anatomy, University of Basel, CH-4056 Basel, Switzerland
}

\begin{abstract}
This study was performed to examine the maintenance of blood vessels in vitro in cortical organotypic slice cultures of mice with special emphasis on basic fibroblast growth factor (FGF-2), which is known to promote angiogenesis and to preserve the integrity of the blood- brain barrier. Slices of neonatal day 3 or 4 mouse brain were maintained for 3, 7, or $10 \mathrm{~d}$ in vitro (DIV) under standard culture conditions or in the presence of FGF-2. Immunohistochemistry for factor VIII-related antigen or laminin revealed a relative low number of blood vessels under standard conditions. In contrast, moderate FGF-2 concentrations increased the number of vessels: with $0.5 \mathrm{ng} / \mathrm{ml}$ FGF-2 it was 1.4-fold higher after DIV 3 or 1.5-fold after DIV 7 compared with controls; with $5 \mathrm{ng} / \mathrm{ml}$ it was almost doubled in both cases. With an excess of $50 \mathrm{ng} / \mathrm{ml}$, FGF-2 vessels were reduced after DIV 3 or similar to controls after DIV 7. FGF receptor 1 was preferentially found on endothelial cells; its immunolabeling was reduced in the presence of the ligand. Cell death detected by an ethidium bromide analog or the apoptosis marker caspase- 3 was barely detectable during the $10 \mathrm{~d}$ culture period. Immunolabeling of the tight junction proteins ZO-1 (zonula occludens protein 1), occludin, claudin-5, and claudin-3 revealed evidence for structural integrity of the bloodbrain barrier in the presence of moderate FGF-2 concentrations. In conclusion, FGF-2 maintains blood vessels in vitro and preserves the composition of the tight junction. Hence, we propose FGF-2-treated organotypic cortical slices as a new tool for mechanistic studies of the blood- brain barrier.
\end{abstract}

Key words: blood- brain barrier; FGF-2; occludin; ZO-1; claudin-3; claudin-5

\section{Introduction}

The blood-brain barrier (BBB) is an endothelial barrier sealed by an extensive network of interendothelial tight junctions (TJs) (Kniesel and Wolburg, 2000). Diseases such as stroke and epilepsy induce disturbances of the BBB with deleterious influences on neuronal function. In vitro models of the BBB employ cultures of dissociated endothelial cells or cocultures with astrocytes. However, the unique BBB phenotype of brain endothelial cells is the result of the continuous influence from the surrounding nervous tissue, including pericytes, perivascular microglia, astrocytes, and the basal lamina, although the details of this interaction are still unclear (Bauer and Bauer, 2000; Abbott, 2002; Haseloff et al., 2005).

The establishment of an in vitro BBB model of cortical organotypic slice cultures (COSCs) that takes the structural connectivity of the cerebral tissue elements into account offers the unique possibility to study single tissue elements under different experimental conditions in the complex environment of the

\footnotetext{
Received April 15, 2006; revised Feb. 15, 2007; accepted Feb. 18, 2007.

We thank M. Saxer and G. Kalt for expert technical assistance and appreciate the expert help of Dr. L. Landmann in confocal microscopy.

Correspondence should be addressed to Dr. Kerstin Bendfeldt at the above address. E-mail: kerstin.bendfeldt@unibas.ch.

DOI:10.1523/JNEUROSCI.4033-06.2007

Copyright $\odot 2007$ Society for Neuroscience $\quad$ 0270-6474/07/273260-08\$15.00/0
}

brain. Compared with conventional BBB models, the blood vessels remain in situ, in a maintained structure that allows cell-cell interactions between natural in vivo partners. We therefore characterized cerebral microvessels and features of the BBB in the presence of basic fibroblast growth factor (FGF-2) in COSCs of newborn mice.

FGF-2 is produced primarily by astrocytes in close proximity to endothelial cells of the BBB (Sobue et al., 1999) and mimics the signaling actions of astrocytes to the BBB (Garberg, 1998, Klint et al., 1999). It binds to FGF receptor 1 (FGFR1) on endothelial cells, exhibits a wide range of angiotrophic effects, and activates signaling pathways involved in regulating endothelial cell survival (Garberg, 1998; Sobue et al., 1999). FGF-2 has also been found to decrease the permeability of the BBB in vitro (El Hafny et al., 1996), which is consistent with the finding that mutant mice lacking FGF-2 show decreased levels of the tight junction proteins occludin and ZO-1 and defects in BBB function (Reuss et al., 2003). Thus, we assumed that FGF-2 might also be a suitable tool to preserve BBB integrity of blood vessels in COSCs of mice and studied its influence on their persistence and structural integrity.

However, blood vessels might perish as a result of trauma caused by explantation and dissection of the slice cultures. Furthermore, the absence of intraluminal blood perfusion might cause regressive changes affecting the $\mathrm{BBB}$ in the course of time. In fact, so far nothing is known about the specific fate of tight 
junction proteins of the BBB in explanted brain tissue, in particular of claudin-5 and claudin-3, which have been shown to be involved in the establishment and maintenance of the BBB in cerebral microvessels of mice (Nitta et al., 2003, Wolburg at al., 2003).

Here, we show for the first time that moderate concentrations of FGF-2 promote maintenance of vascular structures in COSCs of mouse brain and enhance their number in a concentrationdependent manner. It also benefits the maintenance of interendothelial TJs and, thus, BBB function, as indicated by the presence of the tight junction proteins claudin-5, claudin-3, occludin, and ZO-1.

\section{Materials and Methods}

Culture protocol. COSCs were prepared and cultivated according to Stoppini et al. (1991) and Radojevic and Kapfhammer (2004) with slight modifications. Postnatal day 3 (P3) to P4 wild-type mouse pups were rapidly decapitated. After removing the brains, the cortex was roughly dissected together with parts of the underlying striatum or diencephalon, and 350$\mu$ m-thick coronal slices were cut on a tissue chopper. Slices were separated, placed on Millicell-CM $0.4 \mu \mathrm{m}$ culture plates (six slices per membrane; Millipore, Zug, Switzerland) in six-well dishes and were maintained in culture medium, at $37^{\circ} \mathrm{C}$ in a humidified $\mathrm{CO}_{2}$-enriched atmosphere for a minimum of $3 \mathrm{~d}$ to allow tissue recovery from explantation trauma. The medium consisted of HEPES-buffered minimal essential medium (50\%), HBSS (25\%), and heatinactivated horse serum (25\%) complemented with glutamax (2 mM; Invitrogen, Eugene, OR) and adjusted to $\mathrm{pH}$ 7.3.

FGF-2 (Peprotech, London, UK) was added to the medium $(0.5,5$, or $50 \mathrm{ng} / \mathrm{ml}$, respectively) and medium was changed every other day. Control slices were not treated with FGF-2. No antibiotics or antimitotics were used.

Preparation for immunohistochemistry. The cellular differentiation of the slices and the formation of blood vessels was analyzed by immunofluorescence. Cultures were fixed for $2 \mathrm{~h}$ at room temperature (RT) with 4\% PFA in PBS, rinsed three times with $\mathrm{PBS}$, and incubated in blocking solution (BS) containing PBS, 0.5\% Triton X-100, and 3\% normal goat serum for 20 min at RT.

Primary antibodies were diluted in BS as follows: rabbit polyclonal anti-factor-VIII-related antigen (FVIII-rAg; 1:250; Dako, High Wycombe, UK), mouse monoclonal anti FGFR1 (1:250-1:1000; Millipore) rabbit polyclonal anti-laminin (1:75; Sigma, St. Louis, MO), rabbit polyclonal anticaspase-3 (1:250; R \& D Systems, Minneapolis, MN), rabbit polyclonal anti-occludin (1:100; Zymed, San Francisco, CA), rabbit polyclonal antiZO1 (1:100; Zymed), mouse monoclonal anti-claudin-5 (1:50; Zymed), mouse monoclonal anti-claudin-3 (1:100; Zymed), rabbit polyclonal anti-GFAP (1:250), and mouse monoclonal anti-NeuN (1:250) against the DNA-binding neuron-specific protein.

Cultures were incubated overnight at $4^{\circ} \mathrm{C}$, rinsed with PBS at RT, and
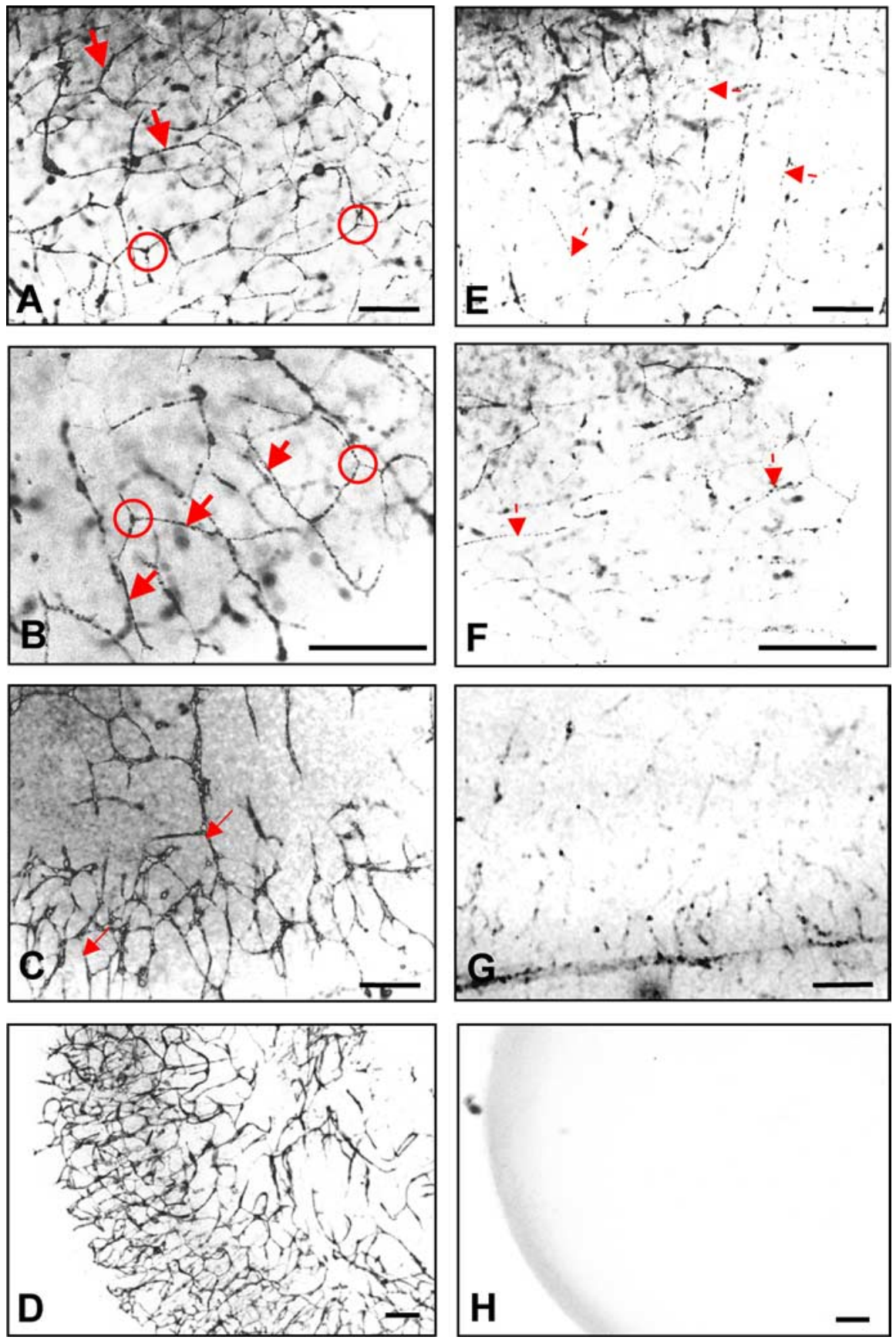

Figure 1. Demonstration of microvessels in neonatal cortical slices under FGF-2 treatments. $A-C$, Immunofluorescence images labeled for FVIII-rAg revealed the presence of microvessels in COSCs after incubation with $5 \mathrm{ng} / \mathrm{ml} \mathrm{FGF-2} \mathrm{at} \mathrm{DIV} 3(\boldsymbol{A})$, DIV $7(\boldsymbol{B})$, and DIV 10 (C). Vessels appear as intact and frequently branching structures with continuous lines (arrows) and intersections (circles). D, Immunostaining for laminin also shows the complexity of the vascular network at DIV 10 after $5 \mathrm{ng} / \mathrm{ml} \mathrm{FGF-2.} \mathrm{E,} \mathrm{F,} \mathrm{Control}$ explants without FGF-2 displayed less labeling for FVIII-rAg at DIV $3(\boldsymbol{E})$ and DIV $7(\boldsymbol{F})$. Note discontinuous arrows marking few discontinuous vascular structures. $\mathbf{G}$, In presence of $50 \mathrm{ng} / \mathrm{ml} \mathrm{FGF-2} \mathrm{at} \mathrm{DIV} 7$ virtually no immunolabeling was observed. $\boldsymbol{H}$, Control slice omitting the first antibody. Scale bars, $100 \mu \mathrm{m}$. incubated with Alexa Fluorochromes (Invitrogen) in BS for $2 \mathrm{~h}$ at RT. Unspecific staining was studied by omitting the primary antibody. The slices were mounted on glass slides with Vectashield mounting medium (Vector Laboratories, Burlingame, CA) and viewed on a Zeiss (Oberkochen, Germany) Axiophot-microscope equipped with an AxiocamHR camera. Recorded images were adjusted for brightness and contrast with Photoshop (Adobe, San Jose, CA).

Confocal microscopy. Fluorescently immunolabeled slices were ana- 


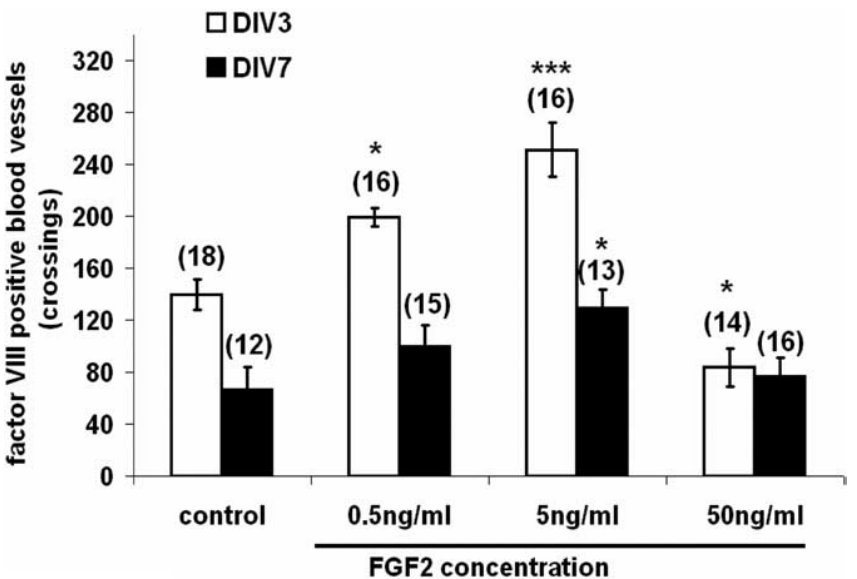

Figure 2. Quantification of vascular segments. Counting of microvessels revealed a relative low number of vessels in controls, which increased in number in a concentration dependent way

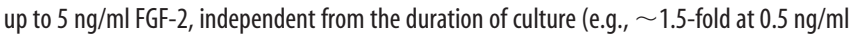
and 2 -fold at $5 \mathrm{ng} / \mathrm{ml}$ ). Excessive amounts of $50 \mathrm{ng} / \mathrm{ml} \mathrm{FGF-2}$ were ineffective in preventing loss of vessels compared with controls. The absolute number of microvessels was halved at DIV 7 compared with DIV 3 . Significances are given versus controls $\left({ }^{*} p \leq 0.05 ;{ }^{* * *} p \leq 0.01\right)$. Error bars indicate SEM.

lyzed using confocal laser scanning microscopy (LSM) (TCS4D; Leica Microsystems, Mannheim, Germany) equipped with an Ar, Kr-laser and an acousto-optical device (acousto-optic tunable filter module). Operating in the simultaneous acquisition mode, images were recorded with an apochromatic $100 \times$ numerical aperture (NA) 1.4 objective lens. After processing with a median filter to suppress background, image stacks were analyzed with the colocalization module of the Imaris software package (Bitplane, Zurich, Switzerland). Images were acquired and reconstructed using the Leica LSM software, cropped, adjusted, and optimized in Photoshop 9.0 (Adobe).

Determination of cell death. Cell death was visualized by application of the fluorescent probe Ethidium-homodimer1 (EthD-1, Life/Dead Kit; Invitrogen). EthD-1 enters cells with damaged membranes and undergoes a 40 -fold enhancement of fluorescence after binding to nucleic acids, thereby producing a bright red fluorescence in nuclei of dead cells. EthD-1 is excluded by the intact plasma membrane of live cells. Apoptosis was visualized by caspase-3 labeling.

Quantification and statistics. The vascular network was quantified according to Moser et al. (2003) with some modifications. The number of vascular crossings was counted in a $6 \times 6$ grid $(100 \times 100 \mu \mathrm{m}$ per single square; total field, $0.25 \mathrm{~mm}^{2}$ ). Recorded images of the cortical slices $(10 \times 0.63$ magnification $)$, were overlaid with three grids per slice in Photoshop and three slices per animal were analyzed. Values in Figure 2 (in brackets) represent the mean of individual slices. Statistical analysis was performed by one-way ANOVA with a subsequent Bonferroni correction as post hoc test ( $p<0.05$ was defined as significant).

\section{Results}

Vessels persist at moderate concentrations of FGF-2

Cerebral microvessels in slices from P3 and P4 mice responded strongly to specific concentrations of FGF-2 exposure as shown by labeling for FVIII-rAg, which is one of the best markers for the identification of endothelial cells and consequently of vascular structures (Fig. 1).

Control slices without FGF- 2 contained a relative low number of FVIII-rAg-positive vessels at $3 \mathrm{~d}$ in vitro (DIV), which further decreased at DIV 7. With moderate FGF-2 concentrations, the number of vessels increased compared with controls: in the presence of $0.5 \mathrm{ng} / \mathrm{ml} \mathrm{FGF-2,} \mathrm{it} \mathrm{increased} \mathrm{1.4-fold} \mathrm{at} \mathrm{DIV} 3$ and 1.5fold after DIV 7; with $5 \mathrm{ng} / \mathrm{ml} \mathrm{FGF-2} \mathrm{it} \mathrm{increased} \mathrm{1.8-fold} \mathrm{or}$ twofold, respectively (Fig. 2). In contrast, with $50 \mathrm{ng} / \mathrm{ml} \mathrm{FGF-2,}$
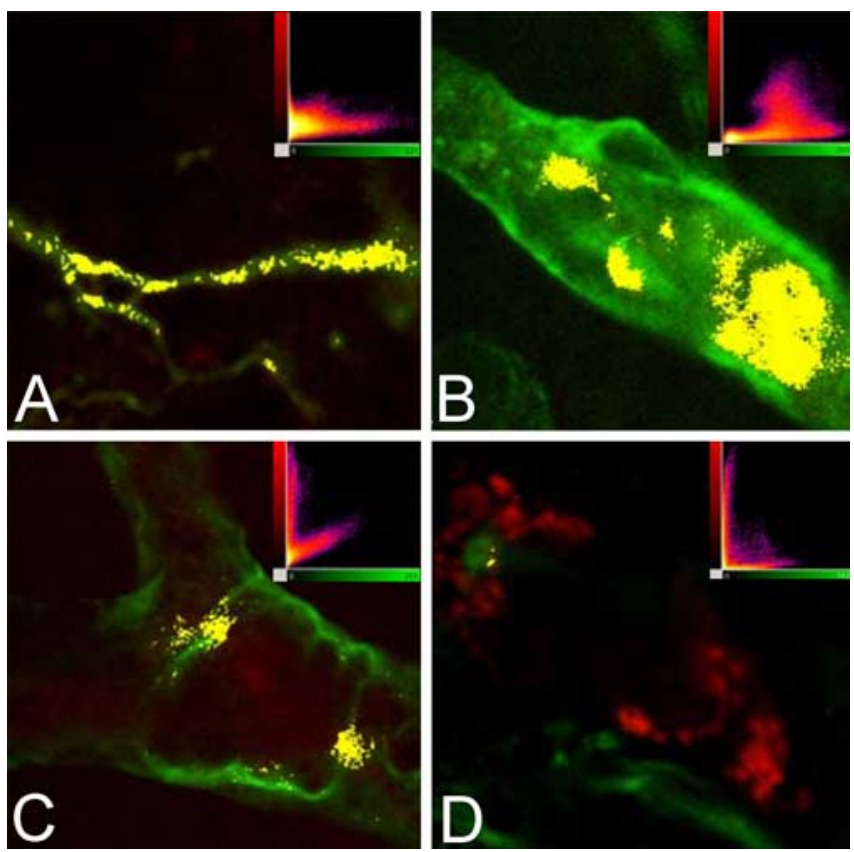

Figure 3. Localization of FGFR1. A-D, Colocalization of FGFR1 and the vascular marker lami$\operatorname{nin}(\boldsymbol{A}-\boldsymbol{C})$ or the astrocyte marker GFAP $(\boldsymbol{D})$ is demonstrated by double immunofluorescence laser confocal microscopy. FGFR1 immunoreactivity is shown in red and laminin or GFAP immunoreactivity are shown in green in these images. Yellow indicates colocalization. Degree of colocalization is also indicated in the diagrams shown as insets in $\boldsymbol{A}-\boldsymbol{D}$. Laminin staining reveals the outline of typical capillaries $(\boldsymbol{A})$ and larger microvessels $(\boldsymbol{B}, \boldsymbol{C})$ at DIV 7 . FGFR1 staining localizes to subregions of the vessels; only little and weak expression of FGFR1 immunoreactivity is found outside of the vasculature. Note the more extensive expression of FGFR1 in controls $(\boldsymbol{A}, \boldsymbol{B})$ compared with FGF-2-treated slices ( $\boldsymbol{C}$. Virtually no colocalization of FGFR1 was seen with astrocytes stained for GFAP (D). All images were recorded with an apochromatic $100 \times N A$ 1.4 objective lens.

the number of vessels decreased 1.7 -fold at DIV 3 compared with controls and remained at control level at DIV 7. Generally, except for the $50 \mathrm{ng} / \mathrm{ml}$ collective, vessels disappear with culture time, $\sim 50 \%$ fewer vessels were found at DIV 7 compared with DIV 3.

Qualitatively, the vascular network was more complex in the presence of 0.5 or $5 \mathrm{ng} / \mathrm{ml} \mathrm{FGF-2.} \mathrm{Vessels} \mathrm{appeared} \mathrm{as} \mathrm{continuous}$ structures after DIV 3 (Fig. $1 A$ ) and DIV 7 (Fig. $1 B$ ). Even at DIV 10, a complex vascular network was observed (Fig. 1C), which was also evident by labeling for the extracellular matrix protein laminin (Fig. 1D). In contrast, without FGF-2, few fragmentary vessels were detected after DIV 3 (Fig. $1 E$ ) and DIV 7 (Fig. $1 F$ ). Virtually no FVIII-rAg-labeled vessels were found in slices treated with an excess of $50 \mathrm{ng} / \mathrm{ml} \mathrm{FGF-2} \mathrm{at} \mathrm{DIV} 7$ (Fig. 1G).

\section{FGFR1 is localized on cerebral vessels}

To show the specificity of the FGF-2 effect on the blood vessels, staining with FGFR1 antibody at DIV 7 was performed together with the vascular marker laminin. Confocal microscopic analysis showed that the laminin staining outlined the blood vessels and that the FGFR1 immunoreactivity was present in localized areas of the blood vessel wall (Fig. $3 A-C$ ). Colocalization analysis (Fig. $3 A-C$, insets) revealed a strong colocalization of the FGFR1 immunoreactivity with the laminin staining. This was the case both for tiny capillaries (Fig. 3A) as for larger vessels (Fig. 3B,C). The immunohistochemical detection of the FGFR1 was more pronounced in the absence of FGF-2 treatment (Fig. 3A,B) compared with the FGF-2-treated slice cultures (Fig. 3C). This reduced immunoreactivity might be attributable to the occupation 

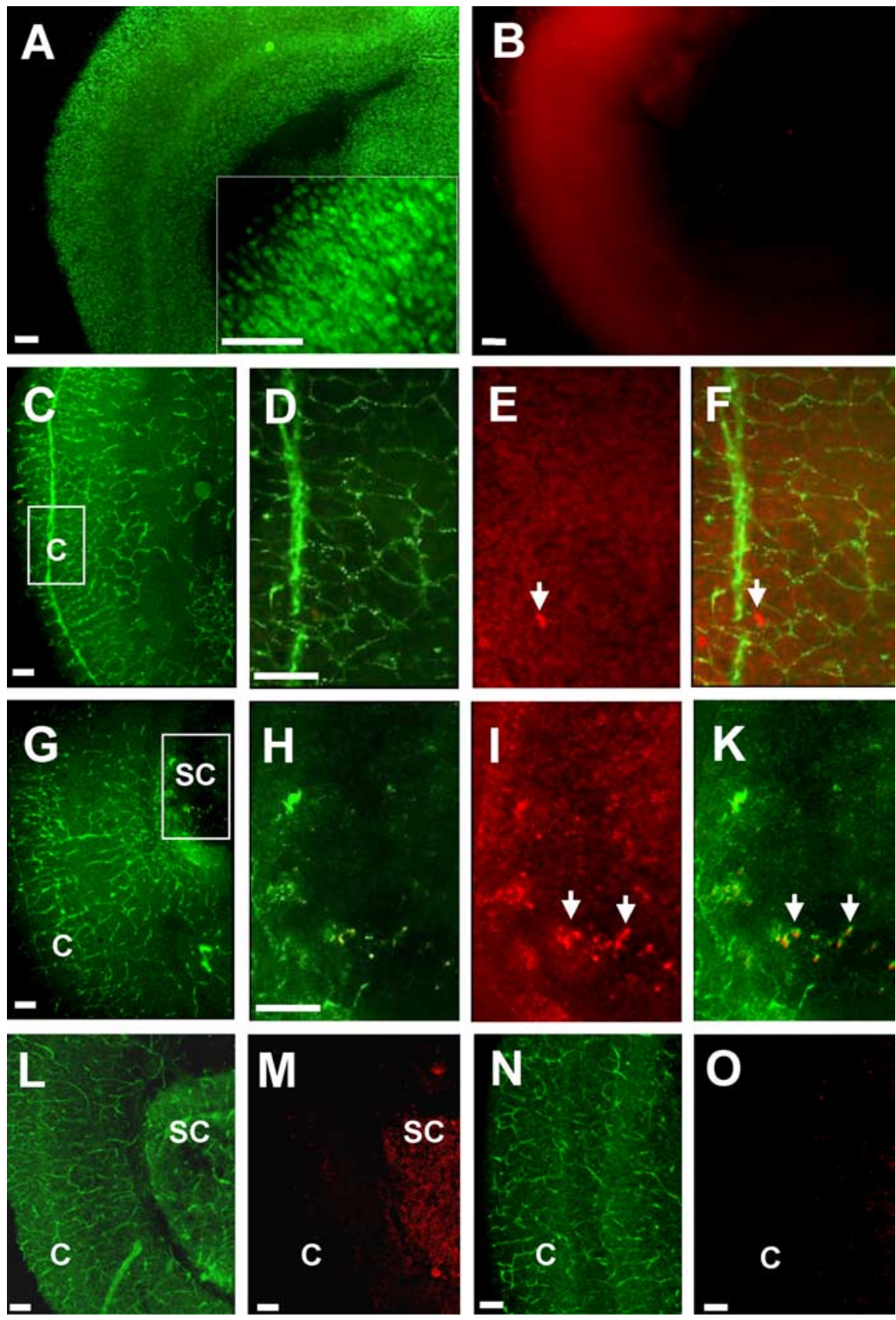

Figure 4. Survival of the slice culture. $A, B$, The organotypic cytoarchitecture of the cortex remained well preserved $(\boldsymbol{A})$ and the overall survival of the tissue was superior in the presence of $5 \mathrm{ng} / \mathrm{ml} \mathrm{FGF-2}(\boldsymbol{B}) . \boldsymbol{A}$, Inset, NeuN labeling of neurons revealed well differentiated cells. $\boldsymbol{B}$, Cell death as shown by EthD-1 staining was barely observed at DIV 7. $\boldsymbol{C}-\boldsymbol{K}$, Images of cortical slices labeled for FVIII-rAg antibody (green) and EthD-1 (red) at DIV 7 (C-F) and DIV $10(\boldsymbol{G}-\boldsymbol{K})$. C, Inset, $\mathbf{D}-\boldsymbol{F}$, Cell death of vascular endothelial cells was not observed in the presence of $5 \mathrm{ng} / \mathrm{ml}$ FGF-2 at DIV 7. Just a few dead cells occurred in the adjacent nervous tissue $\left(\boldsymbol{E}_{\boldsymbol{i}}\right.$ arrows, $\boldsymbol{F}$, overlay). $\boldsymbol{G}$, Inset, $\boldsymbol{H}-\boldsymbol{K}$, At DIV 10, degeneration of the nervous tissue was apparent, but it occurred mainly in the center of the slices ( $\boldsymbol{I}$, arrows, $\boldsymbol{K}$, overlay), indicating the degeneration of neurons and astrocytes rather than endothelial cells of cortical vessels. $\mathbf{L} \mathbf{- 0}$, Apoptotic cell death as shown by caspase-3 staining (red) was nearly absent in the cortical regions of the cultures including cortical blood vessels (laminin staining, green) in the presence of $5 \mathrm{ng} / \mathrm{ml} \mathrm{FGF-2} \mathrm{at} \mathrm{DIV} \mathrm{10.} \mathrm{Apoptotic} \mathrm{degeneration} \mathrm{of} \mathrm{the}$ nervous tissue was observed sometimes in the center of the slices in the subcortical regions. c, Cortical; s.c., subcortical. Scale bars, $100 \mu \mathrm{m}$.

of FGFR1 by its ligand in the FGF-2-treated slices. We also analyzed whether FGFR1 immunoreactivity showed a colocalization with the astrocytic marker GFAP. Virtually no colocalization was seen in astrocytes stained for GFAP (Fig. 3D), indicating that the receptor is expressed mainly by endothelial cells and/or other
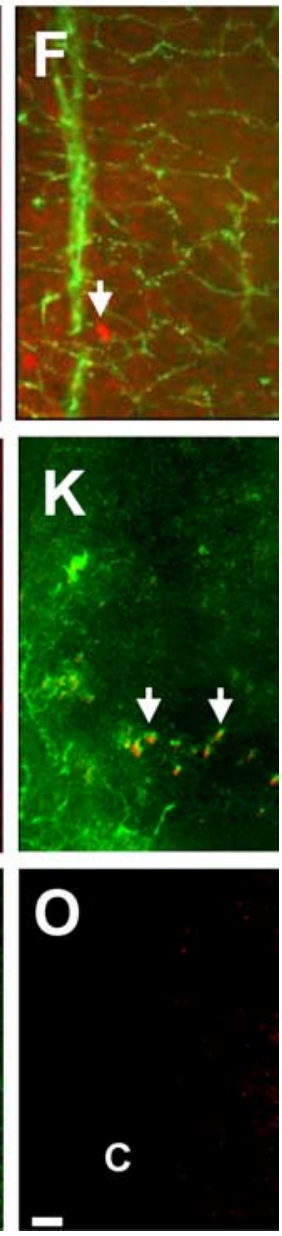

cells of the vascular wall. Together these findings suggest that the endothelial cells of the blood vessel are likely to be the primary target of the FGF-2 treatment.

\section{Vessels persist in a healthy environment}

Because CNS tissue is sensitive to minor environmental variations, it was important to verify whether, in our culture conditions, cell death did occur in the slices, potentially compromising the integrity of the vascular system.

The organotypic cytoarchitecture of the cortex remained well preserved with moderate concentrations of FGF-2 (Fig. 4). Neurons were identified by their immunoreactivity against the DNA-binding, neuron-specific protein NeuN, which is present in most neuronal cell types. Labeling revealed many NeuN-positive cells in the presence of FGF-2 at DIV 7, indicating neuronal differentiation (Fig. 4A). Astrocytes were identified by their immunoreactivity against GFAP, an intermediate filament expressed exclusively in the brain. GFAP labeling at DIV 7 showed evenly distributed, star-shaped astrocytes with multiple branching processes throughout the cortex in untreated controls (Fig. $5 B-D$ ) and in the presence of FGF-2 (Fig. $5 F-K)$. The tips of their vascular processes were plastered along the vessels as shown by double staining of GFAP (green) and FVIII-rAg (red) in the untreated controls (Fig. $5 D$ ) as well as in the presence of FGF-2 (Fig. $5 H-K$ ). However, without FGF-2, most blood vessels have already disappeared at DIV 7 (Fig. $1 F$ ) and the few remaining vascular structures (as shown exemplarily in Fig. 5A) appear extremely dilated.

At moderate concentrations of FGF-2, cell death either of the vascular endothelial cells or of the neighboring cells was barely observed after DIV 7 (Fig. 4B). Cell death was nearly absent in the cortex as visualized by double staining for FVIII-rAg and EthD-1 (Fig. 4E,F), as well as by double staining for laminin and the apoptosismarker caspase-3 (Fig. 4L-O). Just a few dead nuclei were visible (Fig. $4 E, F$, arrows), but a considerable amount of intact microvessels could be shown with FVIIIrAg (Fig. 4C,D). At DIV 10 (Fig. 4G-O), some slices showed signs of beginning degeneration of the nervous tissue in their center (Fig. $4 I, K, M$ ), which was related to the subcortical nervous tissue. However, even at DIV 10, cell death was absent in cortical microvessles (Fig. 4O)

Cell death was also barely detected in controls and in slices treated with $50 \mathrm{ng} / \mathrm{ml} \mathrm{FGF-2} \mathrm{(data} \mathrm{not} \mathrm{shown).} \mathrm{This} \mathrm{might} \mathrm{be}$ attributable to the fact that, under these conditions, most vessels 

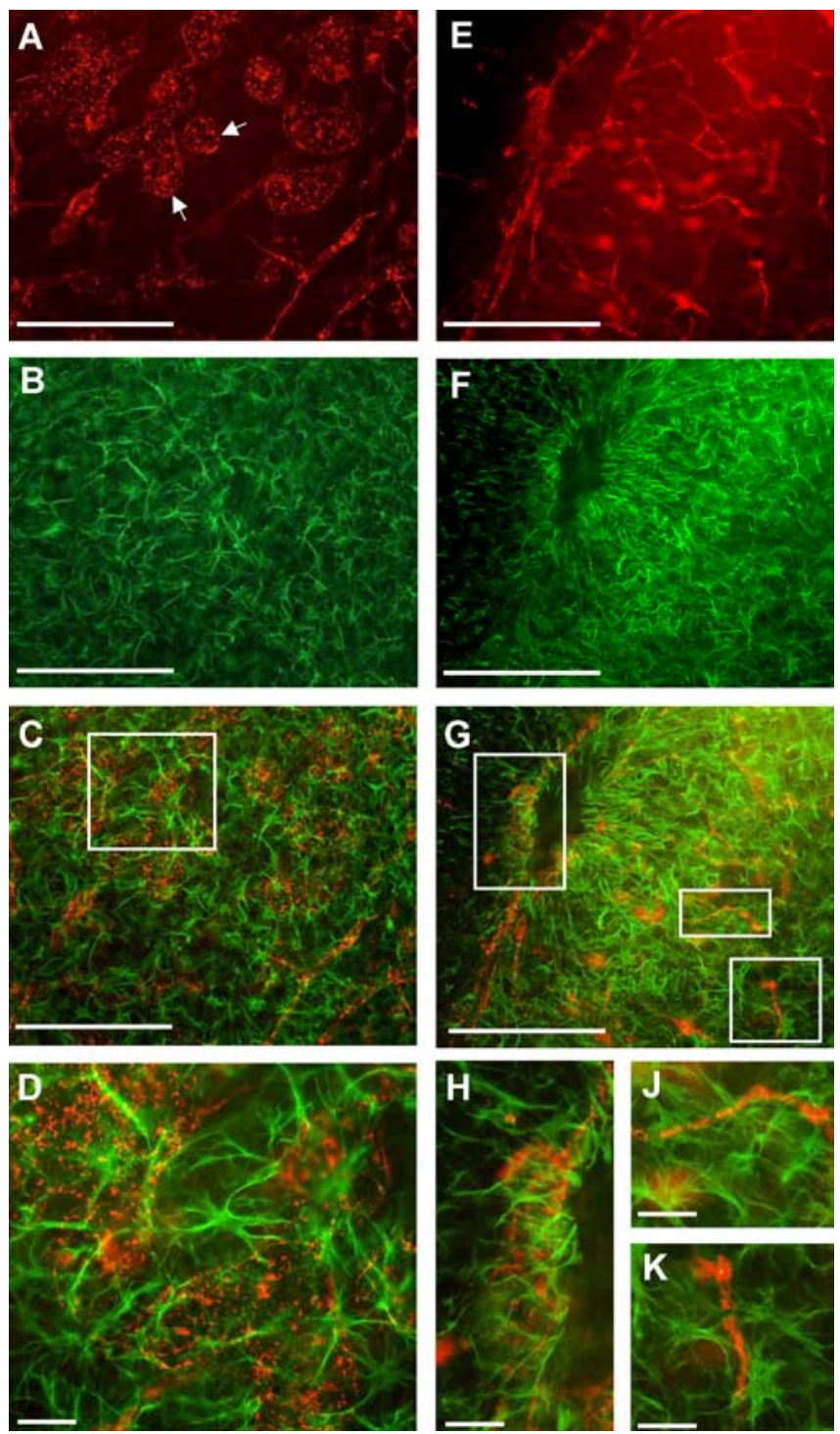

Figure 5. Astrocytes in untreated and FGF-2-treated slices. $\boldsymbol{A}-\boldsymbol{D}$, Untreated controls. $\boldsymbol{E}-\boldsymbol{K}$, FGF-2-treated slices ( $5 \mathrm{ng} / \mathrm{ml}$, DIV 7). $\boldsymbol{A}, \boldsymbol{E}$, FVIII-rAg labeling of vascular structures (red). $\boldsymbol{B}, \boldsymbol{F}$, GFAP labeling of astrocytes (green). Insets in $\boldsymbol{C}$ and $\boldsymbol{G}$ are shown in $\boldsymbol{D}$ and $\boldsymbol{H}-\boldsymbol{K}$. $\boldsymbol{A}$, In the absence of FGF-2, the disintegration of cerebral vessels is apparent (note arrows showing dilated vessels). $\boldsymbol{E}$, In the presence of FGF-2, vascular structures appear intact. $\boldsymbol{B}, \boldsymbol{F}$, GFAP staining shows star-shaped astrocytes, which try to maintain their endfeet around the dilated vascular structures $(\boldsymbol{C}$, inset, $\boldsymbol{D})$. $\boldsymbol{G}$, Insets, $\boldsymbol{H}-\boldsymbol{K}$, In the FGF-2-treated slices, the astrocytic endfeet are in close contact with vessels of different size and shape and surround the blood vessels in a regular manner. Scale bars: $\boldsymbol{A}-\boldsymbol{C}, \boldsymbol{F}, \mathbf{G}, 100 \mu \mathrm{m} ; \boldsymbol{D}, \boldsymbol{H}-\boldsymbol{K}, 25 \mu \mathrm{m}$.

and, thus, degenerated vascular fragments, already had disappeared.

Generally, vessels appear to survive better in explants of younger (P3-P4) pups, compared with older (P5-P7) pups (data not shown), probably because of the better survival of the nervous tissue from younger pups. Thus, for the visualization of the tight junction proteins, P4 animals were used.

Vessels maintain their tight junctions

The microvessels in FGF-2-treated slices kept their in vivo structural integrity as shown by the presence of the tight junction proteins claudin-5 (Fig. 6B-D) (and overlay with laminin F), claudin-3 (Fig. 6G-K), ZO-1 (Fig. 6L), and occludin (Fig. 6M). Their distribution coincided well with the expected localization of tight junction-like structures: continuous, large bands of tight junction proteins were present in the walls of the vascular structures; Figure $6 D-F$ demonstrates exemplarily the localization of claudin-5 and laminin along the vessel walls. Tight junction proteins also regularly delineate adjacent endothelial cells (Fig. $6 \mathrm{~J}, \mathrm{~K}$, arrowheads) as well as the intersections of microvessels $(G, H$, arrows). Thus, in the proposed COSC model, putative regressive changes do not affect tight junction structures in the proposed time-window of culture.

\section{Discussion}

The BBB is challenged in a variety of CNS infectious and neurodegenerative diseases, but most of the studies concerning the $\mathrm{BBB}$ are performed in vivo in animal models, which are not adapted to the study of the underlying mechanistic events. Thus, one of the major issues in $\mathrm{BBB}$ research is the development of representative in vitro models.

Although most in vitro models rely on cultures of dissociated cells, slice cultures offer the unique possibility to study the BBB under different experimental conditions, meanwhile taking into account the complex environment of the brain. Compared with the existing $\mathrm{BBB}$ models, COSCs have the advantage that blood vessels, albeit dissected into smaller fragments, remain in situ in a maintained structure that allows cell-cell interactions between natural in vivo partners.

Although vessels might perish because of the absence of intraluminal blood flow, two early ultrastructural studies in human and newborn rat brain tissue (Wolff et al., 1974; Hauw et al., 1975 ) indicated the survival of vessels in explanted brain tissue. Additionally, more recent immunohistochemical approaches demonstrated the viability of vessels (i.e., in presence of vascular endothelial growth factor in rat cortical cultures) (Kremer et al., 1997; Rosenstein et al., 1998; Moser et al., 2003).

Here, we have taken advantage of FGF-2 as a key molecule in modulating the $\mathrm{BBB}$, promoting angiogenesis and regulating endothelial cell survival (Garberg, 1998; Sobue et al., 1999). Several studies have implicated astrocytes or astrocytic factors in the development and maintenance of $\mathrm{BBB}$ properties in endothelial cells of the brain (Bauer and Bauer, 2000; Garberg, 1998). Among the known astrocyte-derived growth factors, only FGF-2 mimics the signaling actions of astrocytes to the BBB (Klint et al.,1999; Sobue et al., 1999). FGF-2 is a prominent candidate to preserve BBB integrity (Langford et al., 2005), it has been shown to tighten in vitro BBB models (Sobue et al., 1999), and it may be one of the supposed barrier-tightening factors secreted by rat C6 glioma cells (Brown et al., 2003).

Here, we demonstrate for the first time in COSCs of mice that, in presence of $0.5-5 \mathrm{ng} / \mathrm{ml} \mathrm{FGF-2,} \mathrm{vascular} \mathrm{structures} \mathrm{persist} \mathrm{for}$ up to $10 \mathrm{~d}$ while maintaining the structural integrity of the BBB. A correlation between FGF-2 levels and the degree of tumor vascularity was reported in vivo in the CSF of patients with astrocytic brain tumors (Peles et al., 2004). The observed FGF-2 levels, however, were somewhat higher $(20-50 \mathrm{ng} / \mathrm{ml})$ in the clinical study.

Despite the initially lower number of vascular segments in DIV 7 cultures compared with DIV 3 cultures, the increase in the number of vessels after 0.5 and $5 \mathrm{ng} / \mathrm{ml} \mathrm{FGF-2} \mathrm{was} \mathrm{similar} \mathrm{in} \mathrm{both}$ groups (e.g., the number of vascular structures was $\sim 1.5$ higher after $0.5 \mathrm{ng} / \mathrm{ml}$ and doubled after $5 \mathrm{ng} / \mathrm{ml} \mathrm{FGF-2} \mathrm{independent} \mathrm{of}$ the in vitro duration). Whether this is attributable to angiogenesis or an improved survival of already existing blood vessels is not yet clear. The fact that $\sim 50 \%$ of the vessels detectable after DIV 3 were lacking after DIV 7 makes the generation of new vessels rather unlikely. Excessive amounts of $50 \mathrm{ng} / \mathrm{ml} \mathrm{FGF-2} \mathrm{were} \mathrm{also}$ 

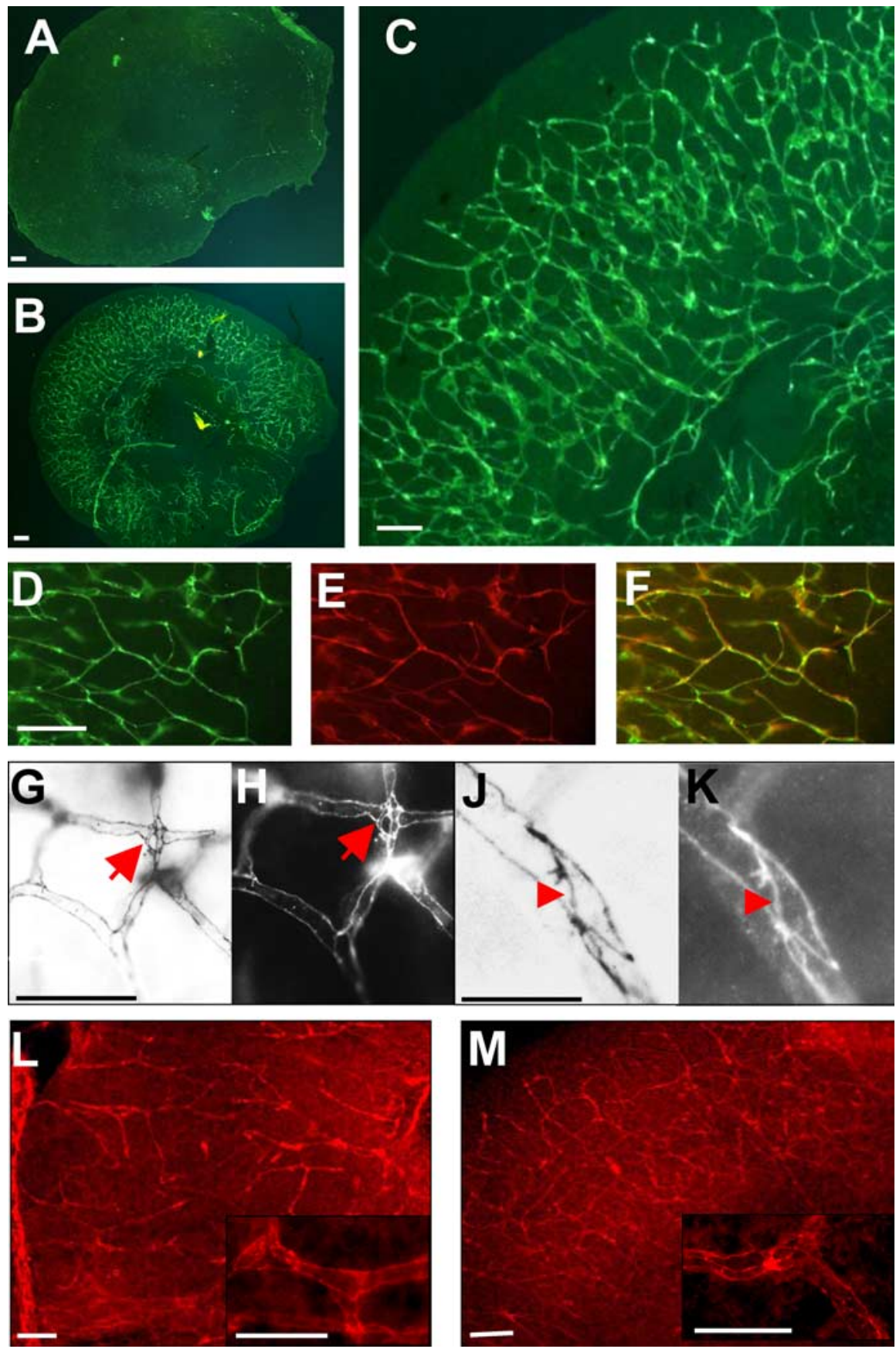

Figure 6. Tight junctions of the BBB appear as intact structures. $\boldsymbol{A}$, In the absence of FGF-2, the loss of tight junction proteins (here exemplarily, claudin-5 labeling) indicates the loss of the integrity of the BBB. $\boldsymbol{B}-\boldsymbol{M}$, Immunostaining revealed a complex network of tight junctions in the slices treated with moderate FGF-2 concentrations (here, $0.5 \mathrm{ng} / \mathrm{ml}$ ) indicating the maintenance of the BBB. Note continuous large bands of claudin-5 (B-D), claudin-3 $(\mathbf{G}-\boldsymbol{K})$, Z0-1 $(\boldsymbol{L})$, and occludin $(\boldsymbol{M})$ delineating vascular walls, intersections between different vessels ( $\boldsymbol{G}, \boldsymbol{H}$, arrows) and adjacent endothelial cells (J, $\boldsymbol{K}$, arrowheads). $\boldsymbol{E}$, Laminin labeling of vessels. $\boldsymbol{F}$, Overlay of laminin and claudin-5. Scale bars, $100 \mu \mathrm{m}$.

ineffective in preventing loss of vessels compared with controls. Thus, FGF-2 in moderate concentrations seems to prolong survival of blood vessels rather then promote angiogenesis.

In the presence of FGF-2, astrocytic endfeet surrounded each individual blood vessel in a regular manner (Simard et al., 2003). In the absence of FGF-2, the disintegration of cerebral vascular structures is obvious. A dilation of vascular fragments occurred at DIV 3 and most vessels were completely disintegrated at DIV 7.
However, even in the absence of FGF-2, astrocytic endfeet tried to maintain contact to the dilated vessels and no differences were found in the microanatomy of astrocytes with or without FGF-2.

Thus, we conclude that, primarily, the vessels themselves and not the astrocytes were affected by the absence of FGF- 2 . This was confirmed by an analysis of the immunohistochemical localization of FGFR1 in COSCs by confocal microscopy. FGFR1 was expressed mainly on blood vessels. A significant amount of the receptor colocalized with laminin, a constituent of the endothelial basal lamina, whereas there was no colocalization with astrocytes. The colocalization of FGFR1 and laminin is in agreement with previous evidence from cell culture experiments that signaling via FGFR1 is dependent on the composition of the extracellular matrix in capillary endothelial cell differentiation, and that laminin plays a pivotal role in these cell-matrix interactions (Kanda et al., 1999).

Survival and functioning of neurons in organotypic slice cultures under control culture conditions in the absence of FGF-2 have been demonstrated by Stoppini et al. (1991), Yamamoto et al. (1992), and many others. We also found a good survival of neurons in the untreated control COSCs for the proposed time-frame (data not shown), as well as in the FGF-2-treated slices.

To exclude that the preservation of blood vessels after FGF-2 treatment might be related to an improved survival of the neuronal and glial elements in the slice cultures, we studied apoptotic cell death with the apoptosis-marker caspase-3. Whereas, in general, caspase- 3 immunoreactivity was absent or very low in the slice cultures, after $10 \mathrm{~d}$ in vitro, some slices showed beginning tissue degeneration in their center. No difference in capase- 3 immunoreactivity was found between control cultures and FGF-2-treated cultures. Furthermore, cell death was related more to the nervous tissue of the subcortical regions and to a much lesser degree to the cortical regions of the slices. Together, these results demonstrate that, in the presence of FGF-2, cerebral blood vessels are well preserved and that this seems not to be caused by an indirect or artificial effect of astrocytes or neurons on the vascular network. However, future studies will clarify the signaling pathways downstream of FGFreceptors and the mechanisms of vascular degeneration in the absence of FGF-2.

It is long established that TJs constitute the anatomical basis of the selective permeability of the BBB (for review, see Wolburg and Lippoldt, 2002). Several peripheral membrane proteins such 
as ZO-1 were reported to concentrate at the cytoplasmic surface of TJs (Schneeberger and Lynch, 1992; Anderson and van Itallie, 1995; Balda and Matter, 1998; Tsukita et al., 1999, 2001) and integral membrane proteins are known to be localized at TJs (i.e., occludin and claudin) (Furuse et al., 1993, 1998).

Claudins are a major constituent of TJ strands (Tsukita et al., 2001), which are copolymers of heterogeneous claudin species (Furuse et al., 1999). Claudin-5 was found specifically in endothelial cells, in large amounts especially in the brain (Morita et al., 1999), leading to the idea that it is directly involved in the establishment of the BBB. Recently, the expression of claudin-3 on in vivo healthy mouse CNS vessels was demonstrated (Wolburg et al., 2003).

Tight junctions seem to play an important role in pathological conditions of the CNS, because alterations in tight junction proteins of the claudin family have been correlated with BBB permeability changes in claudin-5-deficient mice (Nitta et al., 2003) and in leaky blood vessels of dystrophic mdx mice (Nico et al., 2003) as well as during ischemic stroke (Mark and Davis, 2002). The selective loss of claudin-3 immunostaining in tumors or during inflammation suggests that claudin-3 is a central component determining the integrity of BBB TJs (Wolburg et al., 2003).

However, although occludin, claudin-5, and claudin-3 were believed to restrict BBB permeability (Wolburg and Lippoldt, 2002), their expression must not always be correlated with the tightness of the BBB. Removal of astrocytes from cocultures, for example, did not cause any visible loss of tight junction molecules, despite an early BBB opening to paracellular tracers (Hamm et al., 2004). Furthermore, in some neuropathological conditions, the selective loss of claudin-3, but not of claudin-5, ZO-1, or occludin, from altered cerebral vessels indicated that the loss of tight junction proteins might be a rather late event in $\mathrm{BBB}$ breakdown (Wolburg et al., 2003). Yet, this seems to be in contrast to in vivo observations where a complete loss of tight junction proteins was observed in long-established pathological conditions accompanied by BBB breakdown.

An important part of this study was to verify the presence of claudin-3 and claudin-5 in the proposed in vitro model of FGF2-treated COSCs, which suggests well differentiated TJs and the absence of major regressive changes concerning the structural integrity of the BBB. Thus, we propose the COSC-FGF-2 model as a suitable tool for mechanistic studies, especially of late or more pronounced effects on the BBB (i.e., in inflammatory processes during ischemia or neurodegenerative diseases when the adjacent nervous tissue is strongly involved in the pathological event and information on specific cellular interactions and signals leading to BBB breakdown is required). However, the model will also allow study of early and more subtle changes at the BBB distinct from the complete loss of tight junction proteins (e.g., their phosphorylation). Importantly, because of the relative simplicity of the system, COSCs can be readily manipulated and subjected to different insults such as hypoxia, glucose deprivation, and pharmacological treatments to assist in understanding BBB disturbances.

\section{References}

Abbott NJ (2002) Astrocyte-endothelial interactions and the blood-brain barrier permeability. J Anat 200:629-638.

Anderson JM, van Itallie CM (1995) Tight junctions and the molecular basis for regulation of paracellular permeability. Am J Physiol 269:G467-G475. Balda MS, Matter K (1998) Tight junctions. J Cell Sci 111:541-547.

Bauer HC, Bauer H (2000) The blood-brain barrier: Still an enigma? Cell Mol Neurobiol 20:13-29.

Brown RC, Mark KS, Egleton RD, Huber JD, Burroughs AR, Davis TP (2003)
Protection against hypoxia-induced increase in blood-brain barrier permeability: role of tight junction proteins and NFкB. J Cell Sci 116:693-700.

El Hafny B, Bourre JM, Roux F (1996) Synergistic stimulation of gammaglutamyl transpeptidase and alkaline phosphatase activities by retinoic acid and astroglial factors in immortalized rat brain microvessel endothelial cells. J Cell Physiol 167:451-460.

Furuse M, Hirase T, Itoh M, Nagafuchi A, Yonemura S, Tsukita S, Tsukita S (1993) Occludin: a novel integral membrane protein localizing at tight junctions. J Cell Biol 123:1777-1788.

Furuse M, Fujita K, Hiiragi T, Fujimoto K, Tsukita S (1998) Claudin-1 and -2: novel integral membrane proteins localizing at tight junctions with no sequence similarity to occludin. J Cell Biol 141:1539-1550.

Furuse M, Sasaki H, Tsukita S (1999) Manner of interaction of heterogeneous claudin species within and between tight junction strands. J Cell Biol 147:891-903.

Garberg P (1998) In vitro models of the blood-brain barrier. Altern Lab Anim 26:821-847.

Hamm S, Dehouck B, Kraus J, Wolburg-Buchholz K, Wolburg H, Risau W, Cecchelli R, Engelhardt B, Dehouck MP (2004) Astrocyte mediated modulation of blood-brain barrier permeability does not correlate with a loss of tight junction proteins from the cellular contacts. Cell Tissue Res 315:157-166.

Haseloff RF, Blasig IE, Bauer H-C, Bauer H (2005) In search of the astrocytic factor(s) modulating blood-brain barrier functions in brain capillary endothelial cells in vitro. Cell Mol Neurobiol 25:25-39.

Hauw JJ, Berger B, Escourolle R (1975) Ultrastructural observations on human cerebral capillaries in organ culture. Cell Tissue Res 163:133-150.

Kanda S, Tomasini-Johansson B, Klint P, Dixelius J, Rubin K, ClaessonWelsh L (1999) Signaling via fibroblast growth factor receptor-1 is dependent on extracellular matrix in capillary endothelial cell differentiation. Exp Cell Res 248:203-213.

Klint P, Kanda S, Kloog Y, Claesson-Welsh L (1999) Contribution of Src and Ras pathways in FGF-2 induced endothelial cell differentiation. Oncogene 18:3354-3364.

Kniesel U, Wolburg H (2000) Tight junctions of the blood-brain barrier. Cell Mol Neurobiol 20:57-76.

Kremer C, Breier G, Risau W, Plate KH (1997) Up-regulation of flk-1/vascular endothelial growth factor receptor 2 by its ligand in a cerebral slice culture system. Cancer Res 57:3852-3859.

Langford D, Hurford R, Hashimoto M, Digicaylioglu M, Masliah E (2005) Signalling crosstalk in FGF2-mediated protection of endothelial cells from HIV-gp120. BMC Neurosci 6:8.

Mark KS, Davis TP (2002) Cerebral microvascular changes in permeability and tight junctions induced by hypoxia-reoxygenation. Am J Physiol Heart Circ Physiol 282:H1485-H1494.

Morita K, Sasaki H, Furuse M, Tsukita S (1999) Endothelial claudin: claudin-5/TMVCF constitutes tight junction strands in endothelial cells. J Cell Biol 147:185-194.

Moser KV, Schmidt-Kastner R, Hinterhuber H, Humpel C (2003) Brain capillaries and cholinergic neurons persist in organotypic brain slices in the absence of blood flow. Eur J Neurosci 18:85-94.

Nico B, Frigeri A, Nicchia GP, Corsi P, Ribatti D, Quondamatteo F, Herken R, Girolamo F, Marzullo A, Svelto M, Roncali L (2003) Severe alterations of endothelial and glial cells in the blood-brain barrier of dystrophic mdx mice. Glia 42:235-251.

Nitta T, Hata M, Gotoh S, Seo Y, Sasaki H, Hashimoto N, Furuse M, Tsukita S (2003) Size-selective loosening of the blood-brain barrier in claudin5-deficient mice 161:653-660.

Peles E, Lidar Z, Simon AJ, Grossman R, Nass D, Ram Z. Angiogenic factors in the cerebrospinal fluid of patients with astrocytic brain tumors. Neurosurgery 55:562-567.

Radojevic V, Kapfhammer J (2004) Repair of the entorhino-hippocampal projection in vitro. Exp Neurol 188:11-19.

Reuss B, Dono R, Unsicker K (2003) Functions of fibroblast growth factor (FGF)-2 and FGF-5 in astroglial differentiation and blood-brain barrier permeability: evidence from mouse mutants. J Neurosci 23:6404-6412.

Rosenstein JM, Mani N, Silverman WF, Krum JM (1998) Patterns of brain angiogenesis after vascular endothelial growth factor administration in vitro and in vivo. Proc Natl Acad Sci USA 95:7086-7091.

Schneeberger EE, Lynch RD (1992) Structure, function, and regulation of cellular tight junctions. Am J Physiol 262:L647-L661. 
Simard M, Arcuino G, Takano T, Liu QS, Nedergaard M (2003) Signaling at the gliovascular interface. J Neurosci 23:9254-9262.

Sobue K, Yamamoto N, Yoneda K, Hodgson ME, Yamashiro K, Tsuruoka N, Katsuya H, Miura Y, Asai K, Kato T (1999) Induction of blood brain barrier properties in immortalized bovine brain endothelial cells by astrocytic factors. Neurosci Res 35:155-164.

Stoppini L, Buchs PA, Muller D (1991) A simple method for organotypic cultures of nervous tissue. J Neurosci Methods 37:173-182.

Tsukita S, Itoh M, Furuse M (1999) Structural and signaling molecules come together at tight junctions. Curr Opin Cell Biol 11:628-633.

Tsukita S, Furuse M, Itoh M (2001) Multi-functional strands in tight junctions. Nat Rev Mol Cell Biol 2:285-293.
Wolburg H, Lippoldt A (2002) Tight junctions of the blood-brain barrier: development, composition and regulation. Vascul Pharmacol 38:323-337.

Wolburg H, Wolburg-Buchholz K, Kraus J, Rascher-Eggstein G, Liebner S, Hamm S, Duffner F, Grote EH, Risau W, Engelhardt B (2003) Localization of claudin-3 in tight junctions of the blood-brain barrier is selectively lost during experimental autoimmune encephalomyelitis and human glioblastoma multiforme. Acta Neuropathol 105:586-592.

Wolff JR, Rajan KT, Noack W (1974) The fate and fine structure of fragments of blood vessels in CNS tissue culture. Cell Tissue Res 156:89-102.

Yamamoto N, Yamada K, Kurotani T, Toyama K (1992) Laminar specificity of extrinsic cortical connections studied in coculture preparations. Neuron 9:217-228. 